\title{
Time and Space Are Mutually Inherent Property of Each Other, Where the Dependency of Time on Space Can Be Termed as Space-Time and the Dependency of Space on Time Can Be Termed as Time-Space
}

\author{
Prasenjit Debnath
}

PhD Student, NIT Agartala, India

\begin{abstract}
Basically, the physical time and space are mutually inherent property of each other. The analysis of space and the physical time notably started since Euclidean. In the non-relativistic classical theory of mechanics, the usefulness of Euclidean space instead of Einstein's space-time is appropriate, because the physical time is treated as universal property of the Universe with a constant rate of passage that is totally independent of space or the state of motion of an observer. In the relativistic point of view, the physical time cannot be separated from the three dimensional space, because the observed rate at which the physical time passes for a particle or object depends on the object's velocity relative to the observer and also on the strength of gravitational fields, which can slow down the passage of the physical time for an object as observed by the observer outside the field. Thus, in classical mechanics, the physical time and space are mutually independent of each other. A highly successful attempt made by Albert Einstein to combine the three dimensional space with single dimensional time to form a four-dimensional space-time. The concept of space-time combines space and the physical time to a single abstract universe. Mathematically, the space-time is a manifold consisting of "events" which can be described by some type of coordinate system of reference frame. Typically, the three-spatial dimensions (length, width and height), and one temporal dimension (the physical time) are required in the theory of relativity where all dimensions are independent components of the coordinate grid (reference frame) needed to locate a point in a certain defined space. For example, on our globe, the latitude and longitude are two independent coordinates which together uniquely determine any particular location. In Einstein's space-time, a coordinate grid of reference frame that spans the 3+1 dimensions locates events (rather than just points in space), i.e., the physical time is added as another dimension to the coordinate grid of reference frame. This way the coordinate grid of reference frame specifies where and when events occur. In this paper, I will have a different and modified explanation of space-time and I will introduce another new term time-space which is different compared to space-time. The dependency of the physical time on space can be termed as space-time and the dependency of space on the physical time can be termed as time-space. Both space-time and time-space are different as both space and time are two different exposed property of the mystical Universe. They link each other and each of them effects on each other but they are not equivalent at all.
\end{abstract}

Keyword: The non-relativistic classical theory of mechanics, Euclidean space, four-dimensional space-time, coordinate grid of reference frame, the time-space.

\section{Introduction and Theory}

Certainly, three-spatial dimensions (length, width and height) are macroscopic structure of space. The coordinate system of reference frame as described by the theory of special and general relativity is also macroscopic structure of representation of the Universe. Till date, the psychological elemental structure of space is a point $[1,2]$. So, a point is the elemental representation of space $[3,4]$ which does not have any dimension like length, width or height [5]. A point is just a point and it is independent of other points [6, 7]. A unidirectional consecutive collection of points in space can be represented by the macroscopic length dimension and that is used for measurement of distances between points $[8,9]$ in the macroscopic structure of the Universe. A bi-directional collection of points can be represented by two macroscopic dimensions such as length and width to form a surface [10]. Similarly, a three dimensional macroscopic structure (length, width and height) of space represents a volume $[11,12]$. But the macroscopic dimensions are only used for comparative study only (for example, far or near, big surface or small surface, big volume or small volume etc.) and nothing to do with the elemental structure of space such as points which does not have any macroscopic dimension [13]. Thus, macroscopic dimensions are representation of comparative study and only used for measurement purposes. These macroscopic dimensions have nothing to do with the elemental structure of space (point). Every point is independent in space and it has nothing to do with the other points. A huge volume of points provides macroscopic structure of space. Every elemental structure of space (point) is associated with a time having a direction that can be represented as unit vector as reference (the elemental point has its own clock). Because all points are identical in the Universe, they must have their own identical clock. But the macroscopic time of a local area (having symmetry of space points) differs in the macroscopic structure of the Universe. The time runs slow in the Earth compared to Free Space [14, $15]$. We need to adjust the time of satellites with the time on the Earth because in satellite time runs faster compared to Earth $[16,17]$. For each point, if the associated time can be represented as unit vector, then the resultant of these vectors is the macroscopic structure of time for a particular local area (having symmetry of space points) of the Universe such as Earth. For example, the Earth has a resultant vector of 


\section{International Journal of Science and Research (IJSR) \\ ISSN (Online): 2319-7064}

Index Copernicus Value (2013): 6.14 | Impact Factor (2014): 5.611

macroscopic time which runs slower than the resultant macroscopic time of any local area just outside the gravitational attraction of Earth $[18,19]$. Thus, the macroscopic structure of time of any local area (having symmetry of space points) can be different compared to other local area having another symmetry of space points, but the elemental structure of time is a unit vector, and it is identical for every space point. The macroscopic resultant time is different because uneven distribution of space points with different symmetry of unit time in different areas of the Universe. In some limited place of the Universe, the space points are squeezed to form a deformed space which we call it ordinary matter. In some places, the space points can be over relaxed compared to the reference frame to make the resultant macroscopic time of any local area (having symmetry of space points) runs faster than the resultant time of any local area of the reference frame. A huge collection of discrete space points can be so evenly distributed over a huge area of the Universe which will make the macroscopic time of that area identical everywhere in that area. Thus different deformed spaces will have different macroscopic time and the amount of difference is directly proportional to the degree of deformation of space points of a local area (having symmetry of space points). The maximum deformation possible of space at the present state of the Universe is nothing but the Black Holes where matters are in extreme pressure and density possible $[20,21]$. Thus the resultant maximum macroscopic slowness of the physical time is possible in the Black Holes only [22, 23]. The physical time must run really really slow because of extreme deformation of space points in the Black Holes [24, 25].

\section{On the Space Deformations of Different form of Matters}

There are different form of matters such as solid, liquid and gas. And each form has different deformation of space points. In gas form of matter, the space is less deformed or less squeezed than liquid form of matter. Thus the macroscopic time runs faster in a local area of gas than in the local area of liquid form of matter. Similarly, the solid form of matters has higher squeeze or deformation of space compared to the liquid form of matter. Thus in a local area of solid must have slower resultant macroscopic time than the local area of liquid. On psychological point of view, only we can feel macroscopic time of a particle or a body like Earth's macroscopic time, Moon's macroscopic time etc. Even in Earth, there are many different macroscopic resultant times, such as, air is a local area of symmetry of space points in Earth must have different macroscopic time over ocean where ocean is another local area of different symmetry of space points compared to air. Even different solids have different local area of symmetry of space points, thus have different macroscopic resultant physical time.

\section{Every Space Point Has its Own Unit Time Vector}

If the elemental unit of space is a space point $[26,27]$, every point has its own unit time vector. It can be termed as unit time vector of every space point. Any local area that has symmetry of space points can have unique macroscopic time which is nothing but the resultant of all unit vector of time of that local area. A local area must have directional symmetry in time vector to have a unique resultant time vector everywhere in that local area. We can call it a local area having symmetry of space points.

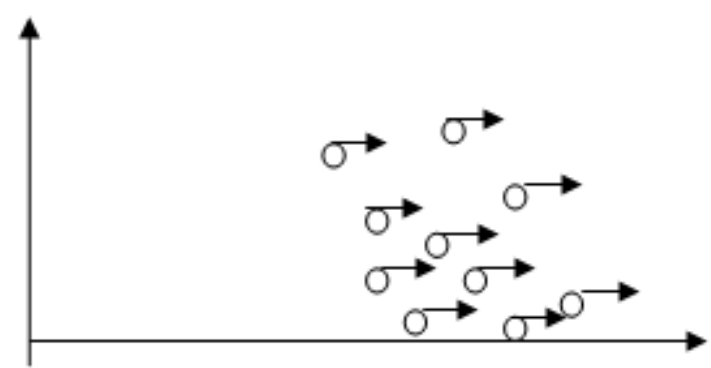

Figure: Each circle is a space point with individual unit time vector with directional symmetry in a local area of the Universe

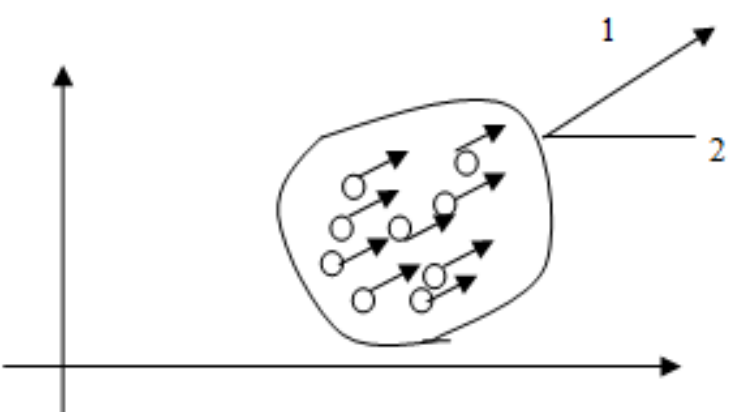

Figure: If the points are more squeezed, the space points forms a local area of different symmetry of space points and the macroscopic resultant vector of time has lower magnitude (2) of horizontal component, thus time runs slower in that local area of symmetry. ( The horizontal component is the macroscopic resultant time can be compared with the time of the reference frame to decide whether time runs slower or faster in that local area).

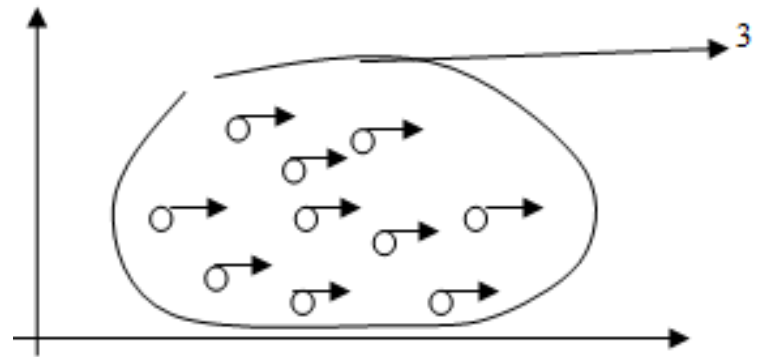

Figure: If the points are over relaxed, the macroscopic resultant vector of time (3) is of higher height as unit time vectors are more close to the parallel with the horizontal line

When space points are squeezed to form ordinary matter, the unit time vectors have different form of symmetry and not parallel with the horizontal time of the reference frame, they must create an angle to reduce the horizontal component of macroscopic time vector somewhat less than the reference frame macroscopic time vector, thus the macroscopic time runs slower there $[28,29]$. As space points are over relaxed, the more relaxed, the more unit vectors of time become parallel with horizontal reference frame, thus the resultant time vector increases more and more. So, there must be highest macroscopic time speed of a local area when all unit 


\section{International Journal of Science and Research (IJSR) \\ ISSN (Online): 2319-7064}

Index Copernicus Value (2013): 6.14 | Impact Factor (2014): 5.611

time vectors are parallel horizontally. Thus the highest speed of macroscopic time can be attained for local area of symmetry of space points.

\section{Conclusion}

The more symmetric the Universe, the faster the macroscopic time will be. If the Universe if infinitely symmetric, we can attain infinite speed of time. There is universal speed limit of macroscopic time and we can assign a number for that speed limit. The space points are discrete and each space point is associated with a unit time vector. The elemental unit of space is a space point which does not have any macroscopic dimension like length, width or height. But certainly, space deformation is the cause of different speeds of macroscopic time to form different local area of symmetry in the Universe. Thus in space-time, space is the cause of time the way it is, just as past is the cause the way the future is. As future is also responsible for the past the way it is and effect is also responsible for the cause the way it is, thus, different times at different local area are also responsible for the way the space points deformation the way it is. Thus as space-time is valid, similarly the timespace is also valid. Psychologically, we understand causeeffect but not effect-cause. The same way, we understand space-time, but not time-space. But both are equally important, mutually dependent. One exists for the other only.

\section{Acknowledgment}

I cordially admire Dr. Aparna Nath, Associate Professor and my PhD Guide, The department of Physics, National Institute of Technology, Agartala, India, for the epitome of inspiration and motivation to write this particular paper with perfection and accuracy. I am extremely thankful to her from all possible help she made to write this paper. Also I am thankful to The Department of Physics of National Institute Of Technology Agartala (NIT Agartala) for proper conduct and coordination.

\section{References}

[1] https://en.wikipedia.org/wiki/Spacetime

[2] Brian Cox, Jeff Forshaw, "The quantum universe: everything that can happen does happen", Penguin Books, pp. 1-44.

[3] Stephen Hawking, "A Briefer History of Time", Bantam Books, London, pp. 1-145.

[4] http://map.gsfc.nasa.gov/universe/uni fate.html

[5] Stephen Hawking, "Black holes and Baby Universes and other essays", Bantam Press, London 2013, ISBN 978-0-553-40663-4

[6] Stephen Hawking, "The Grand Design", Bantam Books, London 2011

[7] Stephen Hawking, "A Brief History of Time", Bantam Books, London 2011, pp. 156-157. ISBN-978-0-55310953-5

[8] Stephen Hawking, "The Universe in a Nutshell", Bantam Press, London 2013, pp. 58-61, 63, 82-85, 9094, 99, 196. ISBN 0-553-80202-X

[9] Stephen Hawking, "The Beginning of Time", A Lecture.
[10] Stephen Hawking, “Stephen Hawking’s Universe: Strange Stuff Explained", PBS site on imaginary time.

[11] Stephen Hawking, "How to build a time machine", 27 April, 2010.

[12] Uno Ingard, K "Fundamental of Waves \& oscillations", Cambridge University Press. P. 38, ISBN-0-521-33957XOxford: The British Academy, 1999

[13]A. Zee, "Quantum Field Theory in a Nutshell", Princeton University Press, 2003

[14] Storrs McCall, "A Model of the Universe", Oxford: Clarendon Press, 1994

[15]Craig Callender, "Time, Reality and Experience", Cambridge, UK: Cambridge University Press.

[16]Craig Callender, "Thermodynamic Asymmetry in Time", The Stanford Encyclopedia of Philosophy (Spring 2002 Edition)

[17] Storrs McCall, "A Model of the Universe", Oxford: Clarendon Press, 1994

[18]Robin Le Poidevin and Murray McBeath, "The Philosophy of Time" Oxford: Oxford University Press, 1993

[19] Newton-Smith, W.H., "The Structure of Time". London: Routledge \& Kegan Paul, 1980.

[20]Barry Dainton,"Time and Space", Ithaca: McGillQueen's University Press, 2001

[21] Robin Le Poidevin, "Questions of Time and Tense", Oxford: Oxford University Press, 1998.

[22] Nerhlich, Graham, "What Spacetime Explains". Cambridge: Cambridge University Press, 1994.

[23] Sklar, Lawrence, "Space, Time, and Space-time". CA: University of California Press, 1974.

[24] Whitrow, G., "The Natural Philosophy of Time". Oxford: Oxford University Press, 1961. (2nd edn., 1980.)

[25] S.W. Hawking, and G.F.R. Ellis, "The Large Scale Structure of Space-Time", Cambridge University Press, (1973).

[26] Stephen Hawking, "A stubbornly persistent illusion-The essential scientific works of Albert Einstein”, Running Press Book Publishers, Philadelphia, London 2011.

[27] Flynn, John L, "Time travel literature", on 29-09-2006

[28] Stephen Hawking, "The Theory of Everything", Jaico Books, pp. 1-110.

[29] Stephen Hawking, "The Illustrated A Brief History of Time”, Bantam Books, pp. 1-21.

\section{Author Profile}

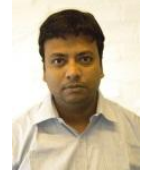

Prasenjit Debnath, born in Agartala, Tripura, India on $15^{\text {th }}$ of March 1979 . He is pursuing PhD in the Department of Physics in National Institute of Technology Agartala (NIT Agartala), India. 